\title{
Interference Coordination for E-MBMS Transmissions in LTE-Advanced
}

\author{
Alberto A. Lopes, ${ }^{1}$ José Seguro, ${ }^{1}$ Paulo Gomes, ${ }^{1}$ Nuno Souto, ${ }^{1,2}$ and Américo Correia ${ }^{1,2}$ \\ ${ }^{1}$ Instituto de Telecomunicações, Av. Rovisco Pais, 1049-001 Lisboa, Portugal \\ ${ }^{2}$ ISCTE-IUL, Av. Das forces Armadas, 1649-026 Lisboa, Portugal
}

Correspondence should be addressed to Américo Correia, americo.correia@iscte.pt

Received 1 April 2010; Accepted 18 August 2010

Academic Editor: Ivan Bajic

Copyright (C) 2010 Alberto A. Lopes et al. This is an open access article distributed under the Creative Commons Attribution License, which permits unrestricted use, distribution, and reproduction in any medium, provided the original work is properly cited.

Interference coordination methods for Evolved-Multimedia Broadcast/Multicast Service (E-MBMS) in Long-Term Evolution Advanced (LTE-A) are presented. In addition, we consider signal space diversity based on Rotation Matrices (RM) known to provide good performance gains over uncorrelated Rayleigh fading channels. OFDM/OFDMA systems can make the use of RM very attractive both for single and multiple antenna transmissions. In this paper, OFDM/OFDMA signals based on LTE parameters are combined with RM, MIMO, Turbo, or LDPC codes. We have considered different types of receivers, namely, we used an MMSE (Minimum Mean Squared Error) equalizer and a Maximum Likelihood Soft Output criterion (MLSO). Frequency, signal, and space diversity gains are evaluated for different spatial channel models (SCM) based on ITU multipath propagation channels. Different adaptive frequency reuse and schedulers are considered to evaluate the E-MBMS spectral efficiency at the cell borders.

\section{Introduction}

Long-Term Evolution Advanced (LTE-A) considers a series of new transmission technologies, such as, coordinated multipoint transmission and reception, relay and carrier aggregation. The objective is to meet the high technical and services requirements of IMT-Advanced standards, such as, peak data rate up to $100 \mathrm{Mbps}$ in high-speed mobility environment and $1 \mathrm{Gbps}$ in a pedestrian environment. The bandwidth varies from $20 \mathrm{MHz}$ to $100 \mathrm{MHz}$. The LTE standard is the basic standard that paves the way for the future 4th Generation (4G) wireless networks.

LTE addresses the emerging trend for the mass provision of rich multimedia services, such as Mobile TV, in a more powerful and spectral efficient way than its predecessors. The Evolved-Multimedia Broadcast/Multicast Service (E-MBMS) framework is envisaged to play an essential role for the LTE-A proliferation in mobile environments. E-MBMS constitutes the evolutionary successor of MBMS, which was introduced in the Release 6 of Universal Mobile Telecommunication System (UMTS). With E-MBMS, the mass provision of multimedia applications to mobile users will be a reality.
With the introduction of Multimedia Broadcast and Multicast Service (MBMS) [1], cellular broadcast will become an essential delivery option of Mobile TV. TV channels can then be broadcasted to an unlimited number of users. On the other hand, the number of channels is limited due to the available broadcast capacity. As MBMS indicates, there are two types of service mode: the broadcast mode and the multicast mode. Each mode has different characteristics in terms of complexity and packet delivery. The broadcast service mode is a unidirectional transmission type. In this service mode, content is delivered, using point-to-multipoint (PTM) transmission, to a specified area without knowing the receivers and whether there is any receiver in the area. In the multicast mode, data is transmitted solely to users that explicitly request such a service. So, there is the possibility for the network to selectively transmit to cells, which contain members of a multicast group. Either point-to-point (PTP) or PTM transmission can be configured in each cell for the multicast operation mode.

PTM transmission does not employ feedback and therefore needs to be statically configured to provide desired coverage in the cell. The transmitted signal is lowest at the cell 


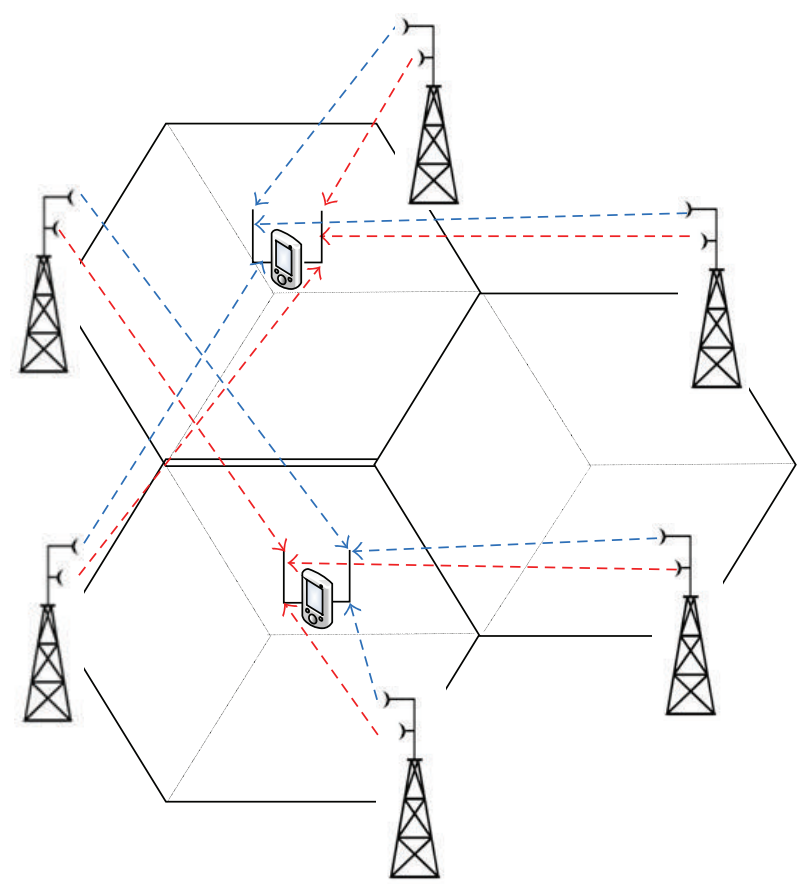

Figure 1: Cooperative MIMO system.

border and therefore the PTM bearer can greatly benefit from exploiting also the signals from adjacent cells transmitting the same service, that is, from soft-combining.

Two types of Evolved-MBMS transmission scenarios exist:

(1) multi-cell transmission (MBSFN: Multi-Media Broadcast over a Single Frequency Network) on a dedicated frequency layer or on a shared frequency layer,

(2) single-cell transmission (SCPTM: Single Cell Point to Multipoint) on a shared frequency layer.

Intercell interference coordination is one method here considered which is expected to improve coverage and increase cell-edge bitrate [2]. Intercell interference coordination techniques, such as reuse schemes and channel allocation, have been studied thoroughly for circuit switched services in second generation accesses [3].

Cooperative MIMO is another emerging technique to combat intercell interference and improve cell edge performance [4]. The system architecture is illustrated in Figure 1.

Sharing data and channel state information among neighboring base stations (BSs) allows to coordinate their transmissions in the downlink and jointly process the received signals in the uplink. Cooperative MIMO techniques can effectively turn intercell interference into useful signals, allowing significant power and diversity gains to be exploited. The architecture of the high-speed backbone enables the exchange of information (data and control information) between the BSs. Cooperative MIMO systems are only concerned with the BS to mobile station (MS) channel which are PTM channels.
The receiver performance of E-MBMS is expected to be improved compared to earlier accesses. Orthogonal Frequency Division Multiple Access (OFDMA) significantly reduces intracell interference compared to MBMS based on CDMA technology. Two receiver antennas are expected to be mandatory in the User Equipment (UE) to mitigate intercell interference. We consider Orthogonal FrequencyDivision Multiplexing/ Orthogonal Frequency Division Multiple Access (OFDM/OFDMA) where the use of Turbo or LDPC codes in combination with multiple input multiple output (MIMO) and signal space diversity is exploited to achieve several gains in band-limited wireless communication systems.

In this paper, we present Turbo and LDPC codes coupled with signal space diversity provided by Rotation Matrices (RM) both Real RM and Complex RM. Both QPSK and M-QAM modulation schemes in several ITU propagation channels will be evaluated starting with a block uncorrelated Rayleigh fading channel. To maximize the diversity order, the constellation of the MQAM signal should be properly rotated such that all distinct symbols are separable on every coordinate.

Two different iterative receivers are introduced to exploit the frequency, signal space, and spatial diversities of OFDM signals with Turbo and LDPC codes, MIMO and RM.

Section 2 introduces the system model and outlines the main blocks of the transmitter and receivers including the signal space diversity provided by RM. The coordinated MIMO and interference schemes are presented in Section 3. Performance curves at link and system level are presented in Section 4, followed by conclusions in Section 5 .

\section{System Model}

Powerful forward error correction (FEC) codes like Turbo and LDPC codes can achieve excellent performances over additive white Gaussian noise (AWGN) channels $[5,6]$. To explore their potential as capacity achieving codes for more realistic wireless channels, we combine them with the use of complex rotation matrices (CRM) [7] and real rotation matrices (RRM) specific to obtain multiresolution with MIMO. RMs provide signal space diversity which can improve the robustness against fading.

Fading causes significant performance degradation in wireless digital communication systems. An optimum design for an AWGN channel does not necessarily result in the best performance in fading channels. Therefore, maximizing the minimum squared Euclidean distance does not necessarily minimize the error probability of this type of channels [8]. Coded modulation techniques coupled with interleaving can improve the performance for block fading channels. It was shown in [9], that for a block fading wireless communication link, diversity can be introduced into the system by separately interleaving the in-phase and quadrature components of a M-QAM scheme and performing symbol-by-symbol detection. It was shown that there was a dependency between the performance of the system and the rotation angle employed in fading channels whereas it was not affected in an AWGN channel. 
2.1. Transmitter. Figure 2 shows the block diagram of an OFDM-MIMO transmitter with RM incorporated. According to the scheme an information block is firstly encoded, interleaved, and mapped onto the constellation symbols. $\mathrm{RM}$ is applied to the resulting sequence by grouping the symbols into size- $M_{\mathrm{CRM}}$ super-symbols and multiplying them by rotation matrix $A_{M_{\text {CRM }}}$. When CRM is employed, matrix $A_{M_{\text {CRM }}}$ belongs to the family of orthonormal complex matrices which, for $M_{\mathrm{CRM}}=2^{n}(n \geq 2)$, are defined as [7]

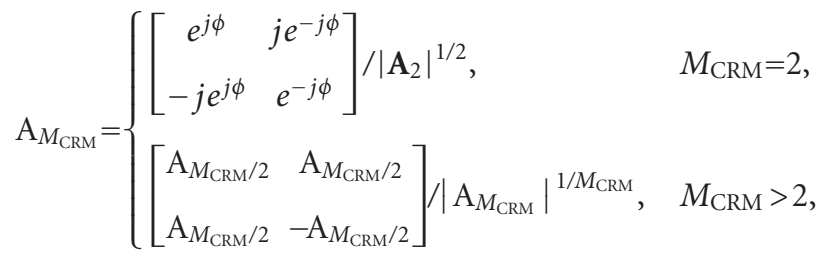

with $\left|\mathbf{A}_{2}\right|=\operatorname{det}\left(\mathbf{A}_{2}\right),\left|\mathrm{A}_{M_{\mathrm{CRM}}}\right|=\operatorname{det}\left(\mathrm{A}_{M_{\mathrm{CRM}}}\right)$, and $\varphi$ being the rotation angle.

A rotated super-symbol can be represented as the vector obtained from

$$
\mathbf{X}=\mathbf{A}_{M_{\mathrm{CRM}}} \cdot \mathbf{S}
$$

where $\mathbf{S}$ is an $M_{\mathrm{CRM}} \times 1$ vector with a set of modulated symbols composing a super-symbol. The resulting sequence is split into $M_{\mathrm{tx}}$ parallel streams which are interleaved in the symbol interleaver. The objective of the symbol interleaver is to explore the characteristics of OFDM transmissions in severe time-dispersive environments whose channel frequency response can change significantly between different subcarriers. Although the different samples are split between the different transmitting antennas, the interleaver insures that samples of a super-symbol transmitted in the same antenna are mapped to distant subcarriers and thus can take advantage of the diversity in the frequency domain. Therefore, the number of transmitting antennas can be lower than the size of the super-symbols, that is, $M_{\mathrm{CRM}} \geq M_{\mathrm{tx}}$. An IDFT (Inverse Discrete Fourier Transform) is applied to the individual sequences, which converts them to the time domain before being transmitted by the respective antennas.

2.2. Receiver. Figure 3 presents the scheme of the receiver employed assuming the use of $N_{\text {rx }}$ receiving antennas. According to the figure, the signal, which is considered to be sampled and with the cyclic prefix removed, is converted to the frequency domain through an appropriate size- $N$ DFT operation and the sequence of symbols is deinterleaved. Assuming that the cyclic prefix is longer than the overall channel impulse response, each received $M_{\mathrm{CRM}}$-sized supersymbol can be represented using matrix notation as

$$
\mathbf{R}=\mathbf{H} \cdot \mathbf{X}+\mathbf{N}
$$

where $\mathbf{H}$ is the frequency response channel matrix. Matrix $\mathbf{H}$ is defined as a blockwise diagonal matrix according to

$$
\mathbf{H}=\left[\begin{array}{ccc}
\mathbf{H}_{1} & & 0 \\
& \ddots & \\
0 & & \mathbf{H}_{M_{\mathrm{CRM}} / M_{\mathrm{tx}}}
\end{array}\right]
$$

with

$$
\mathbf{H}_{k}=\left[\begin{array}{ccc}
H_{k}^{1,1} & \cdots & H_{k}^{1, M_{\mathrm{tx}}} \\
\vdots & \ddots & \vdots \\
H_{k}^{N_{\mathrm{rx}}, 1} & \cdots & H_{k}^{N_{\mathrm{rx}}, M_{\mathrm{tx}}}
\end{array}\right], \quad k=1, \ldots, M_{\mathrm{CRM}} / M_{\mathrm{tx}}
$$

Index $k$ represents a subcarrier position. It is important to note that due to the presence of the symbol interleaver the different sub-carriers denoted by index $k$ may not be necessarily adjacent. To simplify we will assume that $M_{\mathrm{CRM}}$ is a multiple of the number of transmitting antennas $M_{\mathrm{tx}} \cdot \mathrm{N}$ is a $\left(N_{\mathrm{rx}} \cdot M_{\mathrm{CRM}} / M_{\mathrm{tx}}\right) \times 1$ vector containing additive white Gaussian noise (AWGN) samples.

The super-symbol's samples enter the Spatial Demultiplexer and CRM Inverter block which separates the streams transmitted simultaneously by the multiple antennas and inverts the rotation applied at the transmitter. Two alternative methods are employed in this paper: an MMSE (Minimum Mean Squared Error) equalizer [10] and a Maximum Likelihood-based Soft Output (MLSO) detector.

Regarding the first approach, MMSE criterion is applied to each individual subcarrier using $[10,11]$

$$
\hat{\mathbf{X}}_{k}=\left(\mathbf{H}_{k}\right)^{H} \cdot\left[\mathbf{H}_{k}\left(\mathbf{H}_{k}\right)^{H}+\sigma^{2} \mathbf{I}\right]^{-1} \mathbf{R}_{k},
$$

where $\hat{\mathbf{X}}_{k}$ is the $M_{\mathrm{tx}} \times 1$ vector with the estimated subset of coordinates from the super-symbol mapped to sub-carrier $k$, $\mathbf{R}_{k}$ is the $N_{\mathrm{rx}} \times 1$ received signal vector in sub-carrier $k$ with one different receive antenna in each position, and $\sigma^{2}$ is the noise variance. Using the rotated super-symbol estimates $\hat{\mathbf{X}}_{k}$, the component symbol estimates are computed through

$$
\widehat{\mathbf{S}}=\left(\mathbf{A}_{M_{\mathrm{CRM}}}\right)^{-1} \cdot \hat{\mathbf{X}}
$$

In the MLSO criterion, the following estimate is computed for each symbol

$$
\begin{aligned}
\widehat{S}_{l} & =E\left[S_{l} \mid \mathbf{R}\right] \\
& =\sum_{s_{i} \in \Lambda} s_{i} \cdot P\left(S_{l}=s_{i} \mid \mathbf{R}\right) \\
& =\sum_{s_{i} \in \Lambda} s_{i} \cdot \frac{P\left(S_{l}=s_{i}\right)}{p(\mathbf{R})} p\left(\mathbf{R} \mid S_{l}=s_{i}\right),
\end{aligned}
$$

with $s_{i}$ representing a constellation symbol from the modulation alphabet $\Lambda, E[\cdot]$ denoting the expected value, $P(\cdot)$ a discrete probability, and $p(\cdot)$ a probability density function (PDF). Considering equiprobable symbols, we have $P\left(S_{l}=s_{i}\right)=1 / M$, where $M$ is the constellation size. The PDF values required in (8) can be computed as

$$
p\left(\mathbf{R} \mid S_{l}=s_{i}\right)=\frac{1}{M^{M_{\mathrm{CRM}}-1}} \sum_{\mathbf{S}_{l}^{\mathrm{compl}} \in \Lambda^{M_{\mathrm{CRM}}-1}} p\left(\mathbf{R} \mid S_{l}=s_{i}, \mathbf{S}_{l}^{\mathrm{compl}}\right)
$$




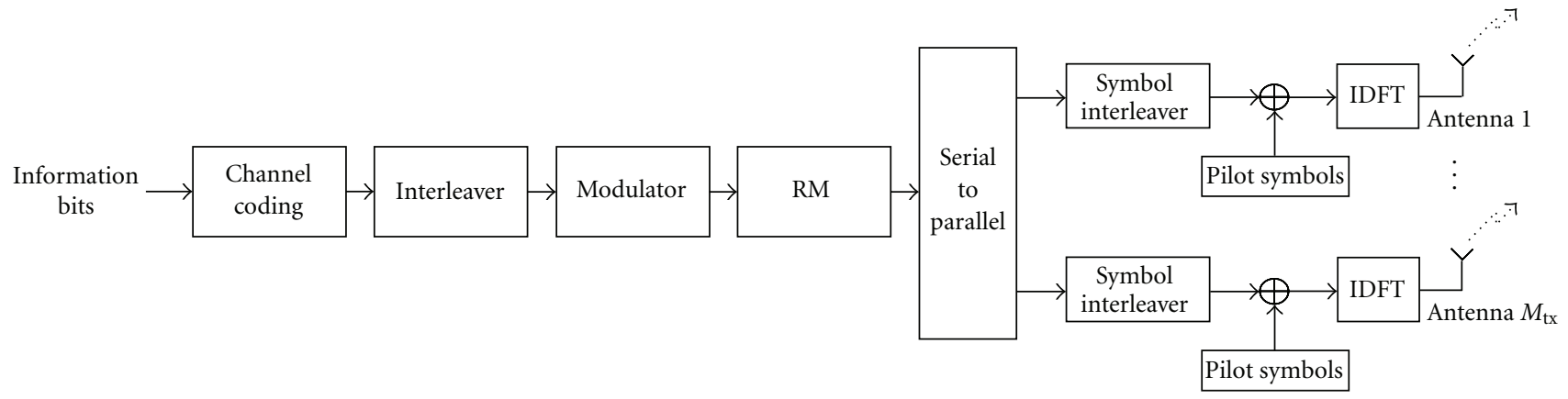

FIgURE 2: Transmitter chain.

with

$$
\begin{aligned}
p\left(\mathbf{R} \mid S_{l}=s_{i}, \mathbf{S}_{l}^{\mathrm{compl}}\right) \\
=\frac{1}{\left(2 \pi \sigma^{2}\right)^{N_{\mathrm{rx}} M_{\mathrm{CRM}} / M_{\mathrm{tx}}}} \\
\quad \times \exp \left[\sum_{n=1}^{N_{\mathrm{rx}} M_{\mathrm{CRM}} / M_{\mathrm{tx}}}-\frac{\left.\mid R_{n}-\mathbf{H}(n,:) \cdot \mathbf{A}_{\left.M_{\mathrm{CRM}} \cdot \mathbf{s}\right|^{2}}^{2 \sigma^{2}}\right],}{}\right.
\end{aligned}
$$

where $\mathbf{S}_{l}^{\mathrm{compl}}$ is an $\left(M_{\mathrm{CRM}^{-1}}\right) \times 1$ vector representing a possible combination of symbols transmitted together with $S_{l}$ in the same super-symbol, $\mathbf{s}$ is an $M_{\mathrm{CRM}} \times 1$ vector comprising $\mathbf{S}_{l}^{\mathrm{compl}}$ and $s_{i}, R_{n}$ is the nth received sample in (3), and $\mathbf{H}(n,:)$ is the nth line of channel matrix $\mathbf{H}$.

Independently of which of the two methods was applied, the resulting symbol estimates are serialized, demodulated, and deinterleaved before entering the channel decoder block which produces the final estimate of the information sequence.

\section{Coordinated MIMO and Interference Schemes}

Without any intercell interference co-ordination, each sector of the cell has unlimited access to the whole bandwidth; this is reuse 1. Any intercell interference co-ordination scheme will restrict the resources available for scheduling, by limiting the (maximum) output power as a function of frequency and/or time, $P_{\max }(f, t)$. We will limit the power $P_{\max }$ both in time (subframe duration) and on frequencies $f$ in a planned scheme on sectors of cells. A pure fractional frequency reuse $1 / 3$ is achieved by dividing the frequencies into three subsets $f_{1}, f_{2}$, and $f_{3}$ and limiting the power by setting

$$
\begin{gathered}
P_{\max }(f, t)=P, \quad f \in f_{n}, \\
P_{\max }(f, t)=0, \quad f \notin f_{n}
\end{gathered}
$$

for each sector of the cell. With reuse partitioning [12], the spectrum is first divided into partitions and then each partition into the desired number of reuse subsets. The scheduler can then utilize the partitions depending on mobile radio position, based on path loss measurements. A reuse partition with a mixture of reuse 1 and $1 / 3$ is achieved by dividing the frequencies into two partitions, $f_{A}$ and $f_{B}$, where $f_{B}$ further is divided into three resulting in four subsets, $f_{A}, f_{B 1}, f_{B 2}$, and $f_{B 3}$. The power limitation for the fractional reuse subsets $f_{\mathrm{Bn}}$ is set as above described.

Soft reuse [13] (hybrid reuse partitioning) is a variant of reuse partitioning where a tighter reuse is achieved by using the same frequencies in more than one partition $\left(f_{A}=f_{B}\right)$ but with different power levels. If we apply to the fractional $1 / 3$ reuse example, then we limit the power by setting

$$
\begin{gathered}
P_{\max }(f, t)=P, \quad f \in f_{n}, \\
P_{\max }(f, t)=p<P, \quad f \notin f_{n} .
\end{gathered}
$$

Figure 4 illustrates the cellular layout (trisectored antenna pattern) indicating the fractional frequency reuse of $1 / 3$ considered in the system level simulations. $1 / 3$ of the available bandwidth was used in each sector to reduce the multicell interference. As indicated in Figure 4, the identification of the sources of multi-cell interference,that is, the use of the same adjacent sub-carriers (named physical resource blocks) is given by the sectors with the same colour, green, yellow, and pink. The small blue hexagons refer to the area where reuse 1 coexists with the fractional reuse of $1 / 3$ as an example of soft reuse.

Figure 1 illustrated the MIMO cooperative system. However, this concept is perfectly applicable to SISO systems. The main feature is the important reduction of intercell interference in the area where the SISO/MIMO cooperative system exists.

In this work, we will consider both techniques to reduce intercell interference and coordinate SISO/MIMO and several reuse portioning schemes.

In the analysis of the scenario Single-Cell Point-toMultipoint (SC-PTM), there is one radio link between the mobile and the closest base station. It does not assume any time synchronism between the transmissions from different base stations with the same colour resulting in interference from all cells without the same colour. However, in the scenario SC-PTM with macrodiversity combining the two best radio links, it is assumed that there is time synchronization between the two closest base station sites with the same colour. In this case the multi-cell interference 


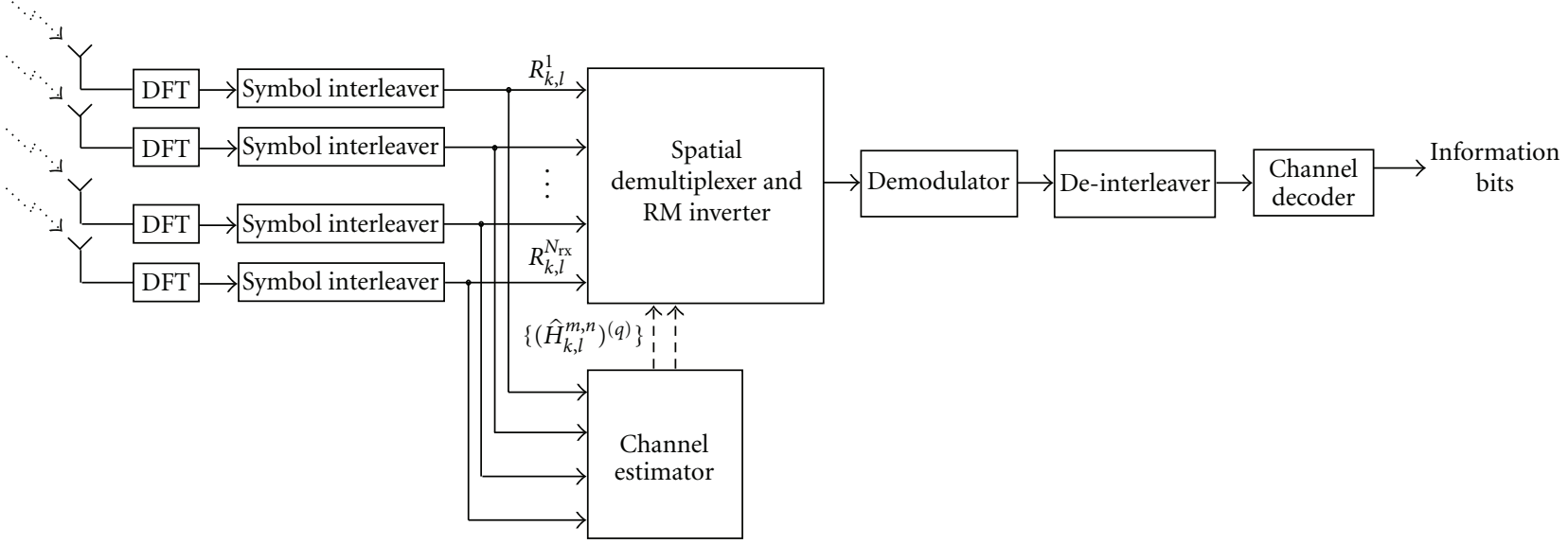

FIGURE 3: Receiver structure.

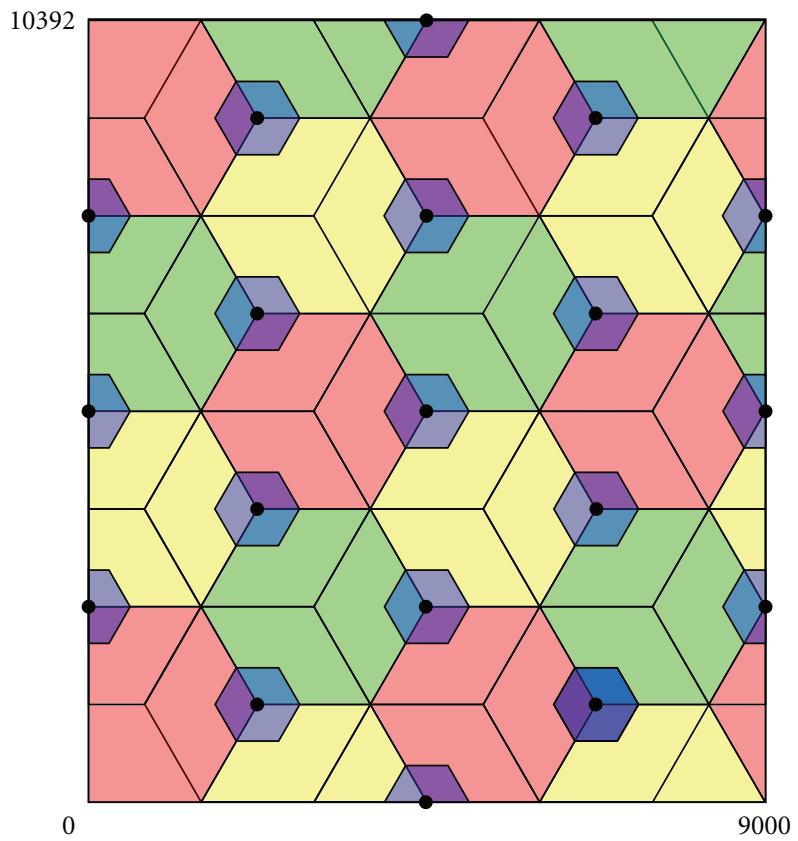

FIgURE 4: Cellular Layout with mixed fractional frequency reuse.

is reduced because only the other base station sites with the same colour remain unsynchronous and capable to interfere.

In the MBSFN scenario, there are at least three radio links, for each of the three closest base stations to the mobile. Time synchronism is assumed between the transmissions from the closest base stations with the same colour resulting in much less interference from the cellular environment. This results in macrodiversity combining of the three best radio links. In addition, the interfering base stations must be at least $5 \mathrm{~km}$ away from the reference base station considering the Cyclic Prefix (CP) long with $16.67 \mu$ s and the frequency of $2 \mathrm{GHz}$. Due to SISO/MIMO coordination, only distant base station sites are capable to introduce interference.
For the purpose of validating the work presented in this section, a system level simulator was developed, using a discrete event-based philosophy, which captures the dynamic behavior of the Radio Access Network System. This dynamic behavior includes the user (e.g., mobility and variable traffic demands), radio interface, and RAN (Radio Access Network) with some level of abstraction. The system level simulator (SLS) works at sub-frame duration rate, and typical time interval of each simulation is 500 seconds. Table 1 shows the simulation parameters. It presents the parameters used in the link and system level simulations based on 3GPP documents [14-16].

\section{Numerical Results}

4.1. BER/BLER Results. To study the behavior of the proposed scheme, several Monte Carlo simulations were performed for the link level simulator. Although this study is valid for any OFDM system, we performed the simulations using the LTE parameters from above-mentioned 3GPP documents and [17] for a $10 \mathrm{MHz}$ bandwidth, which are shown in Table 1.

The channel impulse response is based on several environments from [17] with Rayleigh fading assumed for the different paths. A velocity of $30 \mathrm{~km} / \mathrm{h}$ was considered. The channel encoder was a rate $-1 / 3$ turbo code based on two parallel recursive convolutional codes characterized by $G(\mathrm{D})=[1(1+\mathrm{D} 2+\mathrm{D} 3) /(1+\mathrm{D}+\mathrm{D} 3)][2]$. Puncturing is applied to the parity bits for achieving higher coding rates. At the receiver, a maximum of 12 turbo decoding iterations are applied. The results presented next will be shown as a function of $E_{b} / N_{0}$, where $E_{b}$ is the average information bit energy and $N_{0}$ is the single-sided noise power spectral density.

Figure 5 shows the BER performance of SISO and $2 \times 2$ MIMO of an OFDM transmission in three different ITU channels, namely, the Typical Urban, Flat Rayleigh and Vehicular A channels, with different CRM rotation angles for $E_{b} / N_{0}=8 \mathrm{~dB}$, with turbo coding, coding rate $1 / 2$. The performance seems to not depend on the chosen rotation 
TABLE 1: Simulation parameters for $10 \mathrm{MHz}$ bandwidth.

\begin{tabular}{lc}
\hline Transmission BW & $10 \mathrm{MHz}$ \\
CP length (samples) & 72,256 \\
FFT size & 1024 \\
Number of occupied sub-carriers & 200,600 \\
Sub-frame duration (ms) & 0.5 \\
Sub-carrier spacing (kHz) & 15 \\
OFDM symbols per sub-frame & 6,7 \\
Resource blocks & 50 \\
Distance attenuation ( $d=$ distance in & $\mathrm{L}=122.23+34.88 \log (\mathrm{d})$ \\
kilometers) & $46 \mathrm{dBm}$ \\
Base station power (40 W) & 750,1500 \\
Cell Radius (m) & 3 sectors/site \\
Cell Layout (hexagonal grid) & $\sigma=8 \mathrm{~dB}$ \\
Shadow fading Log-normal & Random walk \\
User Mobility & TypU, MBSFN \\
Multipath fading 3GPP & $15 \mathrm{dBi}$ \\
Max antenna gain (Angular spread & model from SCM, including feeder loss)
\end{tabular}

angle due to the coding rate of $1 / 2$ and the 12 iterations of the turbo decoder. However, for the TypU channel, the effect of the CRM signal space diversity which depends on the chosen angle is observed. According to the results, the Flat channel presents the worst performance followed by the VehA channel and the channel with the best performance is the TypU. This is explained by the increasing number of multipaths and the associated multi-path diversity gain. For all channels, the BER of MIMO transmission is lower than SISO where the carried information bit rate of MIMO is twice of SISO'.

Figure 6 shows the BER performance of a MIMO $2 \times 2$ OFDM transmission in the Flat Rayleigh channel with Turbo codes of coding rate $1 / 2$, two different RM matrices with size 2, a CRM and an RRM. The performance of both MMSE and MLSO receivers is presented. As expected, the performance of MLSO receivers is better than the corresponding MMSE. There is a gain of $1.5 \mathrm{~dB}$ for the $\mathrm{BER}=10^{-2}$. There is no difference in the SSD gain of complex and real matrices as long as they are optimized.

Figure 7 shows the BLER performance of an SISO OFDM transmission in the VehA channel with LDPC codes of different coding rates and two different dimensions of the CRM matrix, $M_{\mathrm{CRM}}=2$ and $M_{\mathrm{CRM}}=16$. The signal space diversity (SSD) gain provided by CRM increases with the size of the CRM matrix but is strongly dependent on the coding rate of the LDPC code. The SSD gain is higher for higher coding rates because of the lower coding gain of these coding rates (less channel bit redundancy). When the redundancy of channel coding increases, the SSD gain is due to the higher diversity gains that channel coding can offer compared to SSD.

Figure 8 corresponds to Figure 7 but the transmission takes place in the TypU channel instead of VehA. Most of the comments presented before regarding the VehA channel are

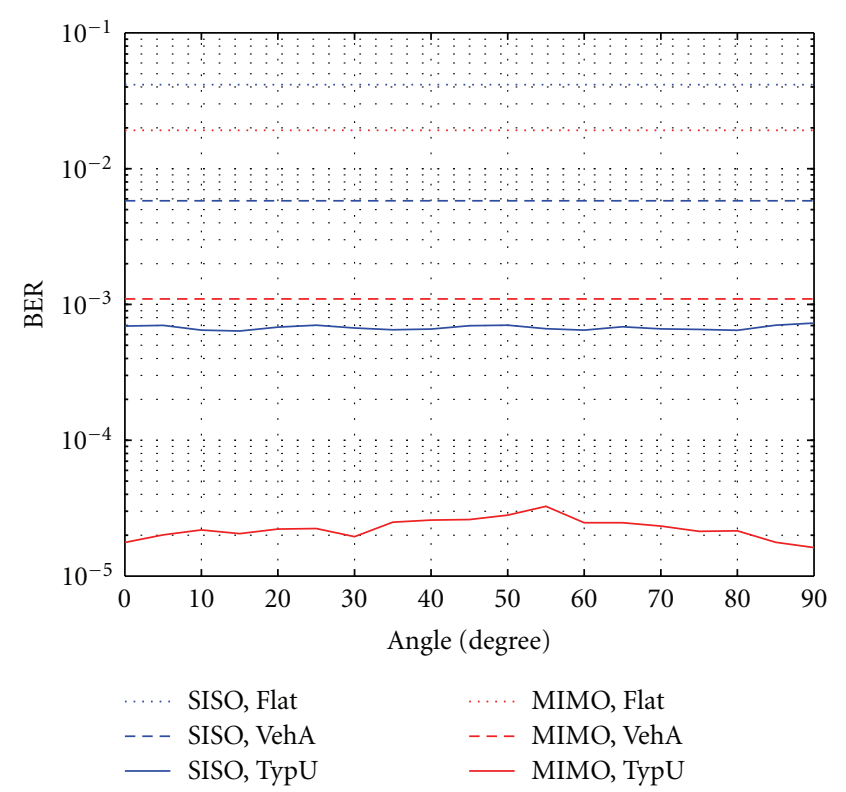

FIGURE 5: BER performance of turbo-coded transmission with CRM2 for different propagation channels.

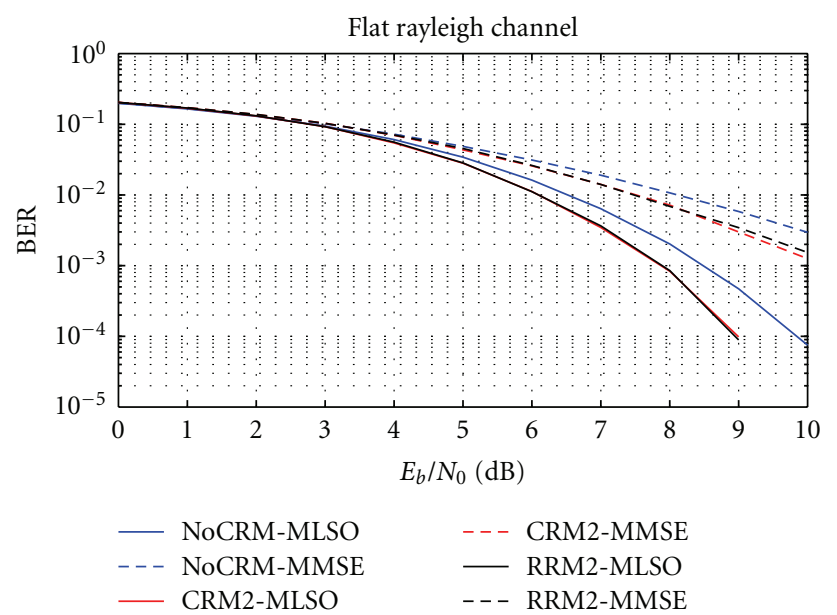

FIGURE 6: BER performance of a MIMO turbo-coded transmission with RM, Flat Rayleigh channel.

also valid for TypU channel. However, the comparison of the two figures indicates that the performance is better for the typical urban channel independently of the channel rate. The gain for BLER $=10^{-2}$ is $2 \mathrm{~dB}$ for both coding rates.

Figure 9 shows the BLER performance of $2 \times 2 \mathrm{MIMO}$ of an OFDM transmission with Hierarchical (H) 16QAM, where the original stream is divided into two QPSK streams, designated as the strong bit blocks and the weak bit blocks. Due to H16QAM and $2 \times 2$ MIMO, the bit stream transmitted by each antenna has blocks four times larger than those of the previous SISO simulations. As expected, the BLER performance of strong blocks is much better than the weak blocks'. According to the performance results for the same signal to noise ratio when strong bit blocks have $\mathrm{BLER}<10^{-3}$, weak bit blocks have BLER $=10^{-1}$. 


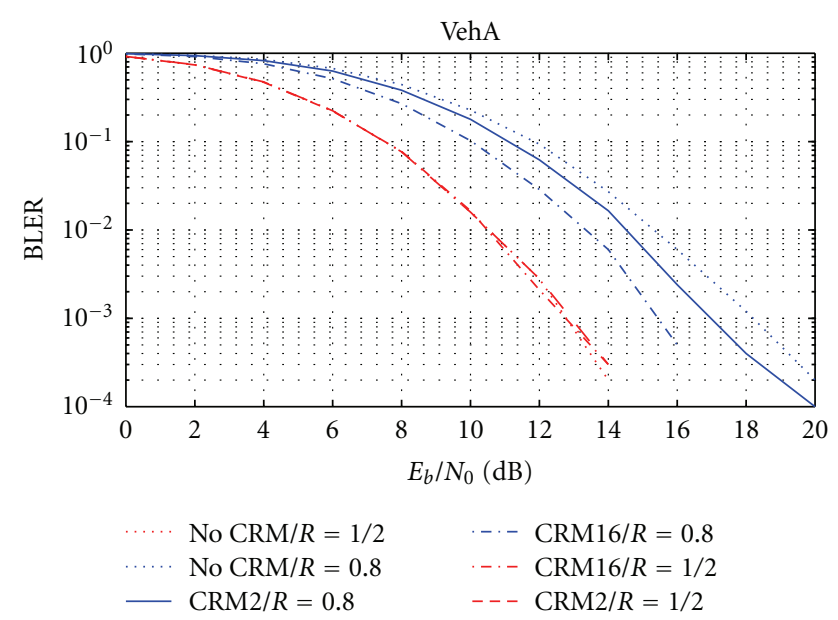

FIGURE 7: BER performance of SISO LDPC coded transmission with CRM, VehA channel.

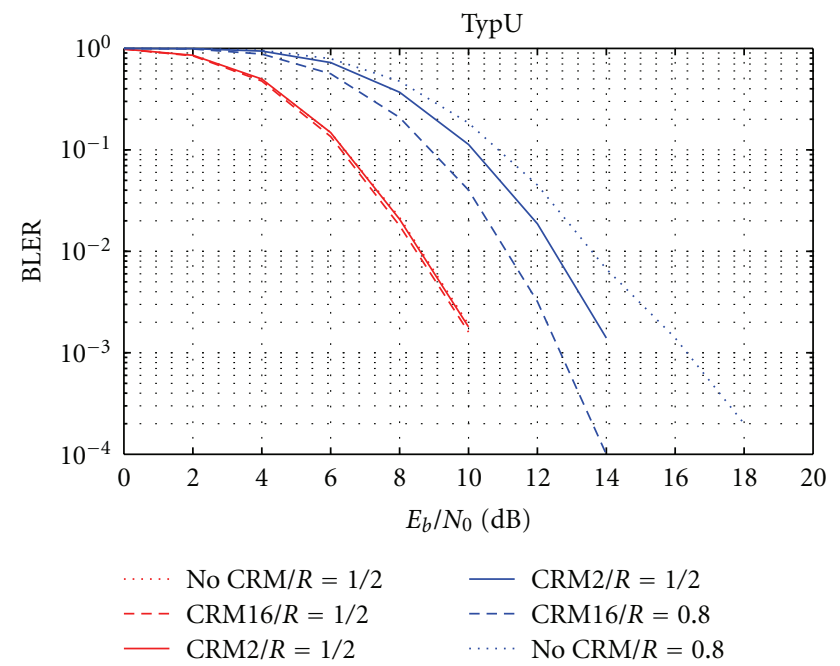

FIGURE 8: BER performance of an SISO LDPC transmission with CRM, TypU channel.

Figure 10 corresponds to Figure 9 but with SSD provided by CRM2, the transmission takes place in the TypU channel, as before. As expected CRM2 provides diversity gain enabling that for weak bit blocks BLER $=10^{-2}$, while for strong bit blocks BLER $<10^{-3}$ keeping the same signal to noise ratio.

4.2. Coverage Results. In the system level simulations mobile users receive blocks of bits transmitted from base stations. Each block undergoes small and large scale fading and multicell interference. In terms of coverage or throughput, the SNR of each block is computed taking into account all the above impairments and based on the comparison between the reference SNR at a BLER of $1 \%$ and the evaluated SNR. it is decided whether the block is or not correctly received. This is done for all the transmitted blocks for all users in all 57 sectors of the 19 cells, during typically 8 minutes and 10 seconds (500 s).

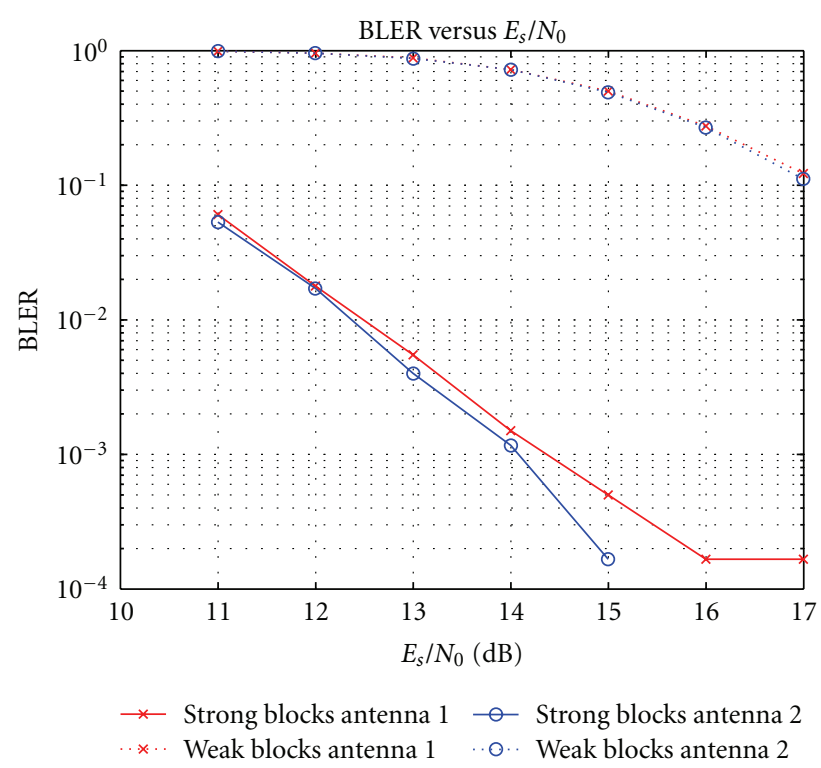

FIGURE 9: BLER performance of $2 \times 2$ MIMO 16QAM turbo-coded transmission without RM, TypU channel.

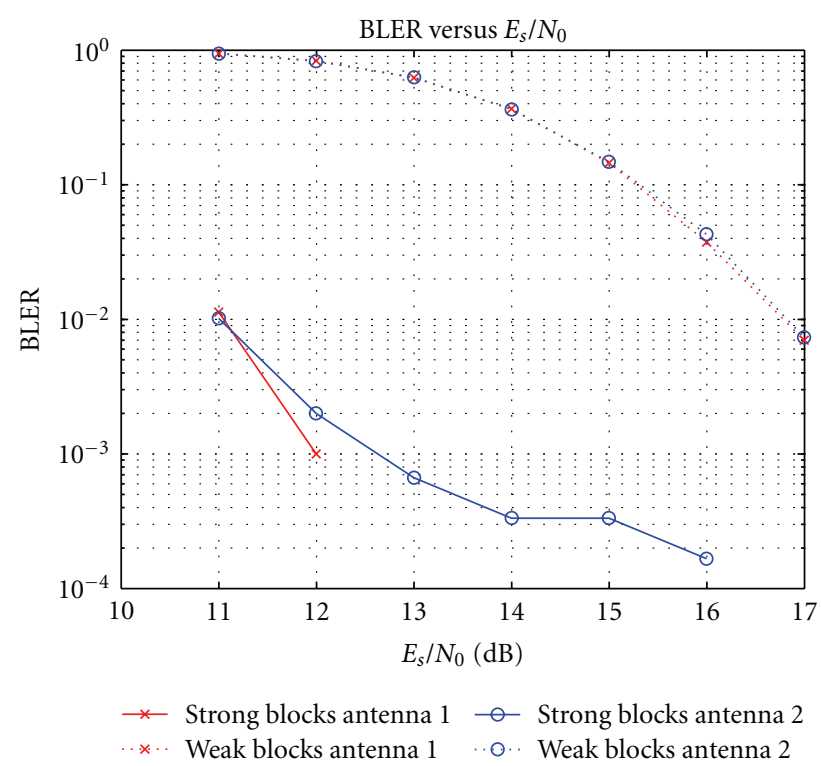

FIGURE 10: BLER performance of $2 \times 2$ MIMO 16QAM turbo-coded transmission with CRM2, TypU channel.

Figure 11 presents the coverage versus the fraction of the total transmitted power (denoted as $E_{c}$ /Ior), for SISO, coding rate $1 / 2$, and SC-PTM scenario, where there is interference only from one third of the sectors due to the frequency reuse of 1/3 (see Figure 4). All interfering sites transmit with the maximum power of $90 \%$ according to the parameters indicated in Table 1 . The cell radius $R$ is $750 \mathrm{~m}$ or $1500 \mathrm{~m}$, and strong blocks (H1) are separated from weak blocks (H2) without any SISO coordination, that is, without macrodiversity combining, denoted as 1RL. In addition to reuse $1 / 3$, reuse 1 and soft reuse (illustrated as hybrid reuse in the legend, consisting of reuse 1 for users inside $D R$ and reuse $1 / 3$ 


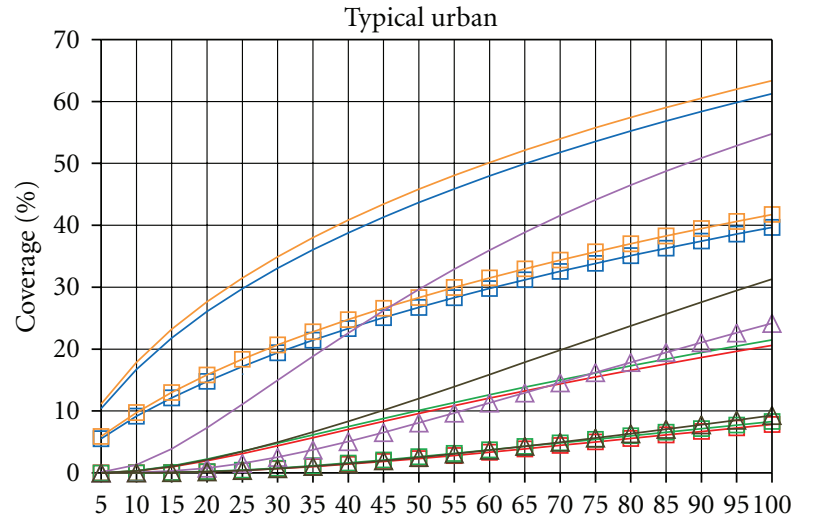

$E_{c} /$ Ior (\%)

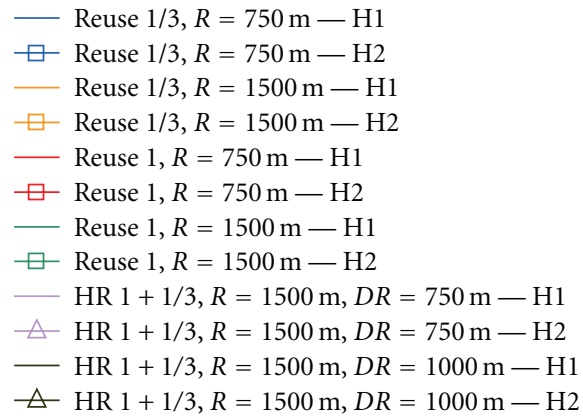

Figure 11: Coverage versus $E_{c} /$ Ior SC-PTM (1RL) scenario.

for users outside $D R$ ) are also evaluated. Reuse 1/3 provides higher coverage followed by soft reuse, the smallest coverage belongs to reuse 1 . However, none reuse scheme assures $95 \%$ coverage.

With macro-diversity combining the two best radio links (2RL), there is a substantial reduction of the multi-cell interference, as indicated in Figure 12. We keep constant the $90 \%$ of maximum transmitted interference power in each site. The only case that never reaches the required coverage is reuse 1 , now both reuse $1 / 3$ and soft reuse with $D R=750 \mathrm{~m}$ assures the required coverage. Remember that the coverage of $\mathrm{H} 1$ is the reference, not the $\mathrm{H} 2$ coverage. According to the coverage results of this figure, we can conclude that with coding rate $1 / 2$ we can increase the cell radius from $750 \mathrm{~m}$ to $1500 \mathrm{~m}$ as long as we include macro-diversity combining of the 2 best radio links for reuse $1 / 3$ and soft reuse with $D R=750 \mathrm{~m}$.

In Figure 13, the coverage performance curves for MBSFN scenario, versus $E_{c} /$ Ior, are presented for both cell radius of $750 \mathrm{~m}$ and $1500 \mathrm{~m}$ and should be compared to the corresponding results of Figure 11 and Figure 12 for the SC-PTM scenario. As expected there is a difference in the coverage between the two scenarios where MBSFN takes advantage of its lower intercell interference. The coverage values for reuse $1 / 3$ are above $95 \%$ even for small values of $E_{c} / N o$ such as $15 \%$; the only exception is the coverage of reuse 1 , independently of the cell radius $R$. There is a coverage similarity between $R=1500 \mathrm{~m}$ and $R=750 \mathrm{~m}$. It means that we should opt to extend the cell radius to decrease
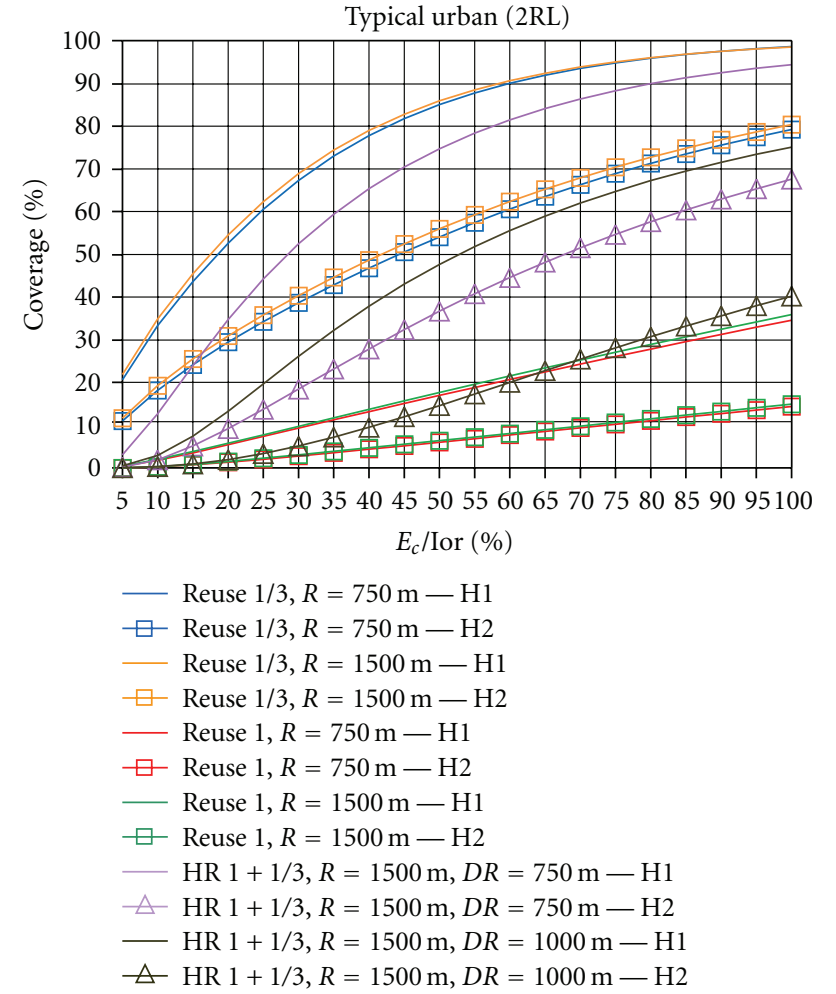

Figure 12: Coverage versus $E_{c} /$ Ior for SC-PTM (2RL) scenario.

the number of cell sites. Both soft reuse cases $D R=750 \mathrm{~m}$ and $1000 \mathrm{~m}$ achieve the reference coverage of $95 \%$, but for different values of $E_{c}$ /Ior. When we increase the distance $D R$ of soft reuse from $750 \mathrm{~m}$ to $1000 \mathrm{~m}$, we are increasing the $E_{c}$ /Ior and the capacity (the average throughput) in the cell. To increase the throughput we opt to decrease the coverage of, in particular, $\mathrm{H} 2$ blocks.

Another important technique is the use of spatial multiplexing (MIMO) associated to signal space diversity (SSD) provided by CRM to enhance the capacity. The spectral efficiency of QPSK, $2 \times 2$ MIMO, is equivalent to H16QAM with SISO illustrated in previous figures. Figure 14 presents the coverage versus the fraction of the total transmitted power, for coding rate $1 / 2$ and SC-PTM scenario. Instead of $\mathrm{H} 1$ and $\mathrm{H} 2$ blocks, now we have Antenna 1 (A1) and Antenna 2 (A2) blocks, where the coverage of each antenna is about the same. The cell radius $\mathrm{R}$ is again $750 \mathrm{~m}$ or $1500 \mathrm{~m}$, and we consider the existence of coordinated MIMO transmission, that is, with macro-diversity combining the two best radio links. In addition to reuse $1 / 3$, reuse 1 is also evaluated. As expected, the coverage of reuse $1 / 3$ is higher than the reuse 1 due to less intercell interference. It does not matter the cell radius of the coverage is the same, which indicated that we should choose the higher cell radius to reduce the number of sites.

Figure 15 corresponds to Figure 14; the difference is the coding rate that is $3 / 4$. For reuse $1 / 3$, the reference coverage of $95 \%$ is achieved as before independently of the cell radius. Due to the higher spectral efficiency, there is a decrease of 


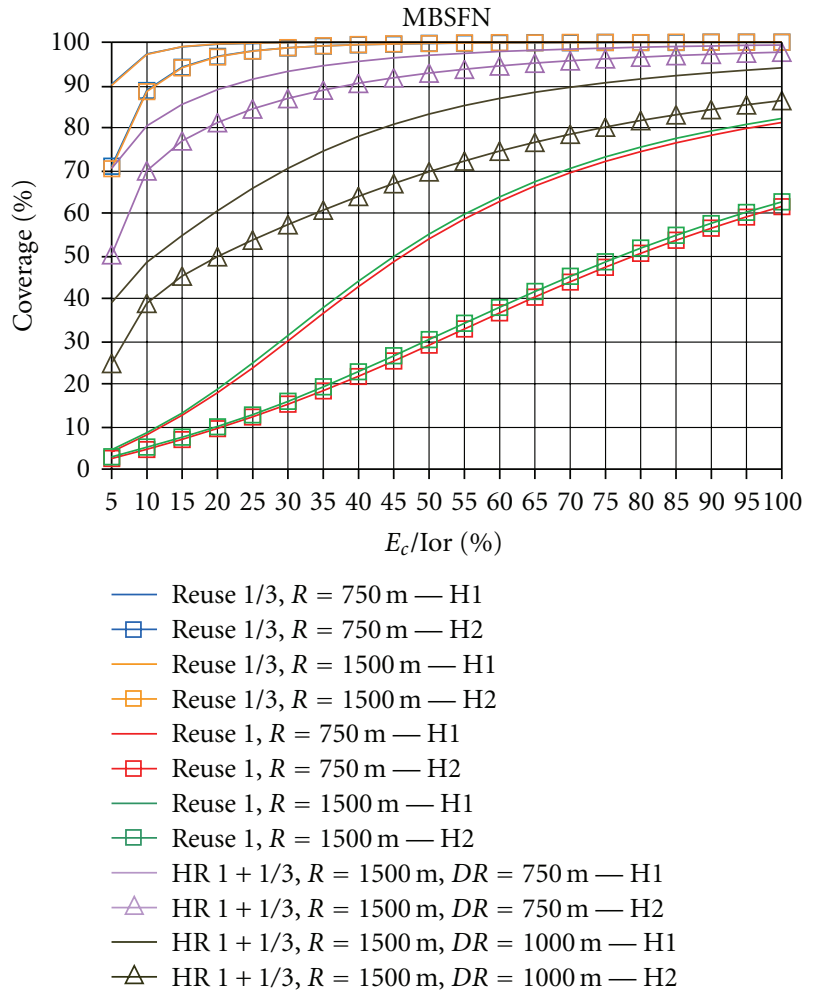

Figure 13: Average coverage (\%) versus $E_{c} /$ Ior (\%), of MBSFN.

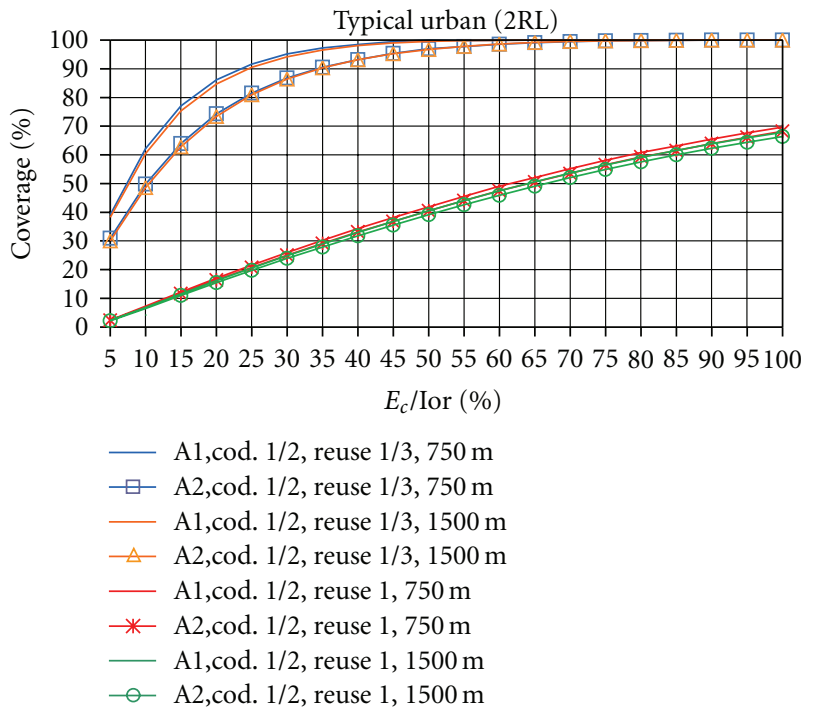

Figure 14: MIMO coverage (\%) versus $E_{c} /$ Ior (\%), for SC-PTM scenario, cod. $1 / 2$.

coverage. However, the reduction of coverage due to the higher coding rate is not substantial, which should lead to higher throughput for high values of $E_{c} /$ Ior.

4.3. Throughput Results. Figure 16 presents the average throughput distribution as a function of the $E_{c}$ /Ior for coding rate $1 / 2$ and the SC-PTM scenario with macro-diversity

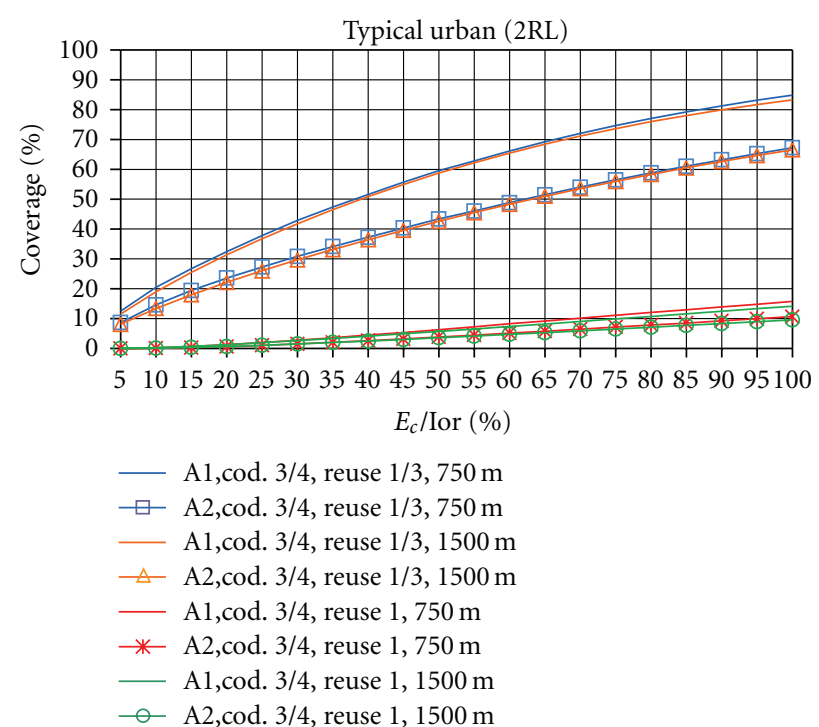

Figure 15: MIMO coverage (\%) versus $E_{c} /$ Ior (\%), for SC-PTM scenario, cod. $3 / 4$.

combining for both cell radii of $750 \mathrm{~m}$ and $1500 \mathrm{~m}$ and different reuse schemes. We observe that the maximum throughput is achieved for soft reuse with $D R=1000 \mathrm{~m}$ followed by reuse 1 for both cell radii. In spite of the smaller coverage, reuse 1 achieves an average throughput higher than the throughput of reuse $1 / 3$ due to its higher throughput for users located close to the base station with small intercell interference. However, it is necessary to transmit more than $65 \%$ of the total transmitted power to have higher average throughput than the achieved by reuse 1/3. But, not all UEs are able to achieve such high throughput since users located at the cell borders never reach such high values of throughput. So soft reuse is the best compromise in terms of maximum average throughput.

Figure 17 corresponds to previous figure but considering the MBSFN scenario. We observe that now the maximum throughput is achieved for reuse 1, which was already expected due to its inherent maximum capacity associated to less intercell interference provided by the SISO coordination of the MBSFN network. However, it is necessary to transmit more than $45 \%$ of the total transmitted power to obtain higher average throughput for reuse 1. Even that not all UEs are able to achieve the maximum throughput, in particular, users located at the cell borders, it is recommended to choose reuse 1 associated with the MBSFN network.

To increase the spectral efficiency at the cell borders, we will check the use of $2 \times 2$ MIMO associated with QPSK modulation and SSD provided by CRM. Figure 18 presents the average throughput versus $E_{c}$ /Ior for coding rate $1 / 2$ and the SC-PTM scenario with macro-diversity combining the 2 best radio links. We observe that the maximum throughput is achieved for reuse 1 and reaches $11000 \mathrm{kbps}$, which is the same value of the MBSFN scenario with H16QAM presented in Figure 17. This confirms the higher spectral efficiency of MIMO compared to H16QAM independently of the chosen reuse scheme. 


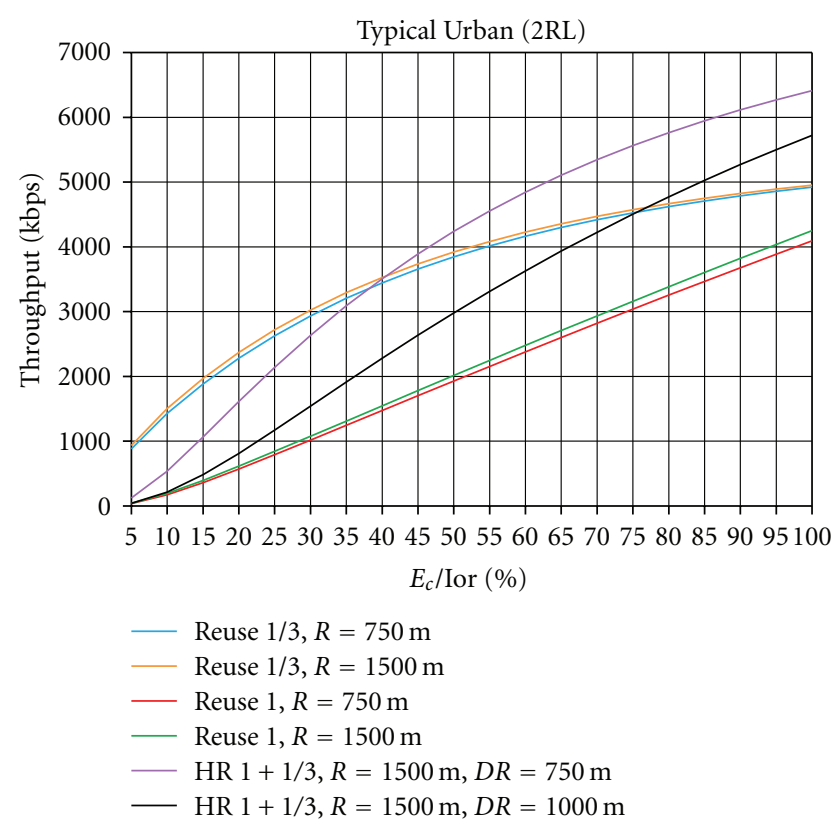

FIgURE 16: Throughput versus $E_{c}$ /Ior for SC-PTM (2RL) scenario.

Figure 19 corresponds to Figure 18, the difference is the coding rate of $3 / 4$. As expected for reuse 1 with the highest fraction of transmitted power, the throughput reaches $12400 \mathrm{kbps}$. In spite of the lower coverage, the average throughput is higher with coding rate $3 / 4$, but it does not reach 1.5 times the throughput of coding rate $1 / 2$. This confirms that with coding rate $3 / 4$, users at the cell borders have less throughput than those that are located closer to the BS.

To increase further the spectral efficiency at cell borders, it is suggested to keep the coding rate of $3 / 4$ and use the coordinated MIMO transmissions provided by the MBSFN network.

\section{Conclusions}

In this paper, we have analyzed interference coordination methods for Evolved-Multimedia Broadcast/Multicast Service (E-MBMS) in Long-Term Evolution Advanced (LTEA). In addition, we introduce signal space diversity based on Rotation Matrices (RM) known to provide good performance gains over uncorrelated Rayleigh fading channels. OFDM/OFDMA chosen for E-MBMS with the use of RM and both single and multiple antenna transmissions are associated with Turbo or LDPC codes. We have considered an MMSE (Minimum Mean Squared Error) equalizer, and a Maximum Likelihood Soft Output criterion (MLSO) as possible receivers.

We conclude that for higher coding rates SISO OFDM systems, the introduction of RM provides signal space diversity gain, for both Turbo and LDPC codes that increase with the size of the rotation matrices, independently of the rotation angle chosen. For lower coding rates, the

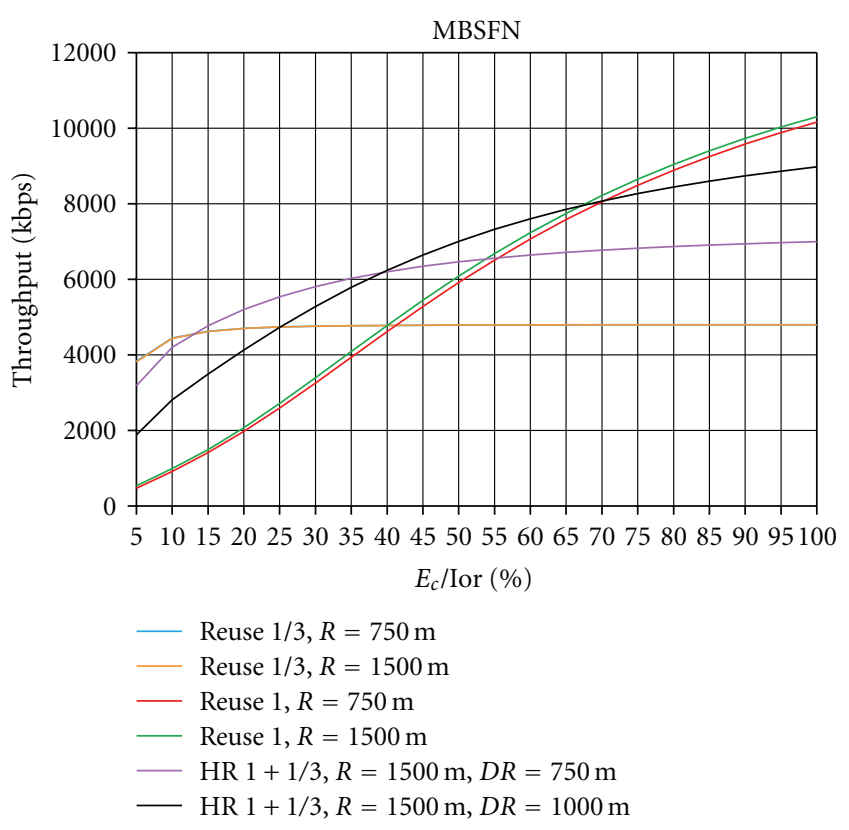

FIgURE 17: Throughput versus $E_{c} /$ Ior for MBSFN scenario.

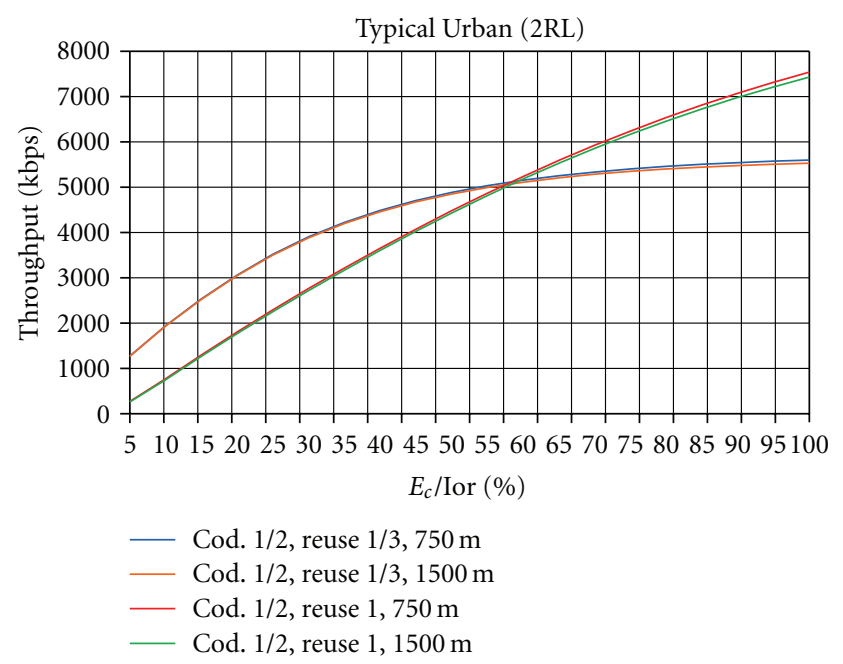

FIGURE 18: MIMO Throughput versus $E_{c} /$ Ior for SC-PTM, cod. 1/2.

introduction of RM only provides diversity gain for turbo codes and is smaller than with higher coding rates.

Based on the average coverage and throughput results, for the SC-PTM scenario, the use of soft reuse, that is, a mixture of reuse 1 for users closer to BS and reuse $1 / 3$ for users at the cell borders is recommended. It is also suggested to apply some coordination between the point-to-multipoint transmissions of adjacent BSs. For the MBSFN scenario, we recommend the use of reuse 1 due to its best compromise between coverage and maximum achieved throughput.

The introduction of signal space diversity and spatial multiplexing $2 \times 2 \mathrm{MIMO}$ enables enhancing the spectral efficiency at the cell borders of SC-PTM. Further enhancement of the spectral efficiency is possible increasing the coding 


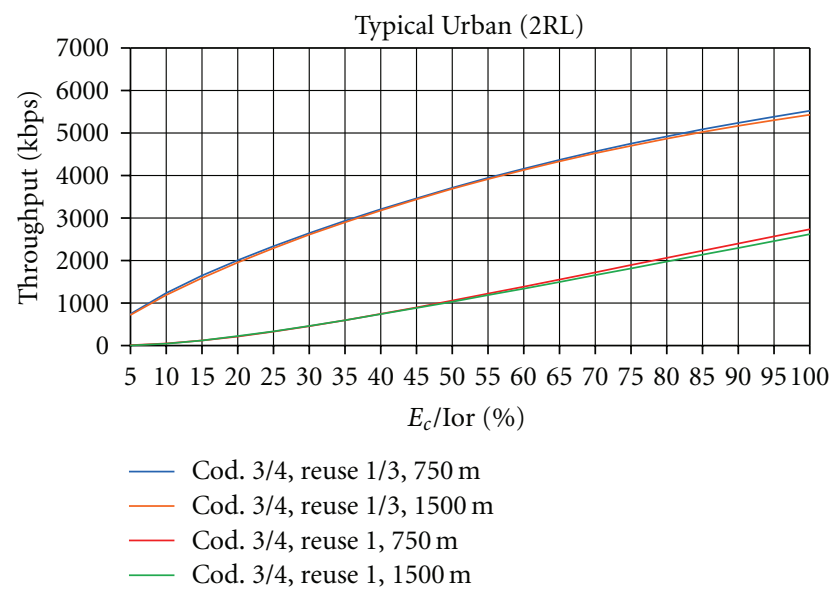

Figure 19: MIMO Throughput versus $E_{c} /$ Ior for SC-PTM, cod. 3/4.

rate within the MBSFN network where coordinated MIMO transmissions occur.

\section{Acknowledgment}

This work was partially supported by the FCT - Fundação para a Ciência e Tecnologia (pluriannual funding, U-BOAT project PTDC/EEA-TEL/67066/2006) and the internal IT project designated as COILS.

\section{References}

[1] 3GPP, "Feasibility Study on Improvement of the Multimedia Broadcast Multicast Service (MBMS)," TR 25.905 version 7.2.0 Release 7, http://www.3gpp.org.

[2] 3GPP, "Physical Layer Aspects for Evolved UTRA," TR 25.814,V1.2.2, 2006.

[3] I. Katzela and M. Naghshineh, "Channel assignment schemes for cellular mobile telecommunication systems: a comprehensive survey," IEEE Personal Communications, vol. 3, no. 3, pp. 10-31, 1996.

[4] C.-X. Wang, X. Hong, X. Ge, X. Cheng, G. Zhang, and J. Thompson, "Cooperative MIMO channel models: a survey," IEEE Communications Magazine, vol. 48, no. 2, pp. 80-87, 2010.

[5] C. Berrou and A. Glavieux, "Near optimum error correcting coding and decoding: turbo-codes," IEEE Transactions on Communications, vol. 44, no. 10, pp. 1261-1271, 1996.

[6] T. J. Richardson, M. A. Shokrollahi, and R. L. Urbanke, "Design of capacity-approaching irregular low-density paritycheck codes," IEEE Transactions on Information Theory, vol. 47, no. 2, pp. 619-637, 2001.

[7] A. Correia, "Optimised complex constellations for transmitter diversity," Wireless Personal Communications, vol. 20, no. 3, pp. 267-284, 2002.

[8] C. Schlegel and D. J. Costello Jr., "Bandwidth efficient coding for fading channels: code construction and performance analysis," IEEE Journal on Selected Areas in Communications, vol. 7, no. 9, pp. 1356-1368, 1989.

[9] J. Boutros and E. Viterbo, "Signal space diversity: a power- and bandwidth-efficient diversity technique for the rayleigh fading channel," IEEE Transactions on Information Theory, vol. 44, no. 4, pp. 1453-1467, 1998.
[10] S. M. Kay, Fundamentals of Statistical Signal Processing: Estimation Theory, Prentice-Hall, Englewood Cliffs, NJ, USA, 1993.

[11] N. Souto, J. C. Silva, R. Dinis, F. Cercas, and A. Correia, "An iterative receiver for WCDMA systems with MIMO transmissions and hierarchical constellations," in Proceedings of the IEEE International Symposium on Spread Spectrum Techniques and Applications (ISSSTA '06), pp. 233-237, Manaus, Brazil, August 2006.

[12] T. Salvalaggio, "On the application of reuse partitioning," in Proceedings of the 38th IEEE Vehicular Technology Conference (VTC'88), pp. 182-185, 1988.

[13] G. Fodor, "Performance analysis of a reuse partitioning technique for OFDM based evolved UTRA," in Proceedings of the 14th IEEE International Workshop on Quality of Service (IWQoS '06), pp. 112-120, June 2006.

[14] ETSI, "Selection procedures for the choice of radio transmission technologies of UMTS," ETSI, TR 101112 v3.2.0, Cedex, France, 1998.

[15] 3GPP, 25.212-v6.2.0, "Multiplexing and Channel Coding (FDD)".

[16] 3GPP, "Feasibility study for evolved Universal Terrestrial Radio Access (UTRA) and Universal Terrestrial Radio Access Network (UTRAN),” Tech. Rep. 25.912 v7.1.0.

[17] 3GPP, "Evolved-UTRA Radio Frequency (RF) System Scenarios,” TR. 36.942 v9.2.0 (2009-12). 

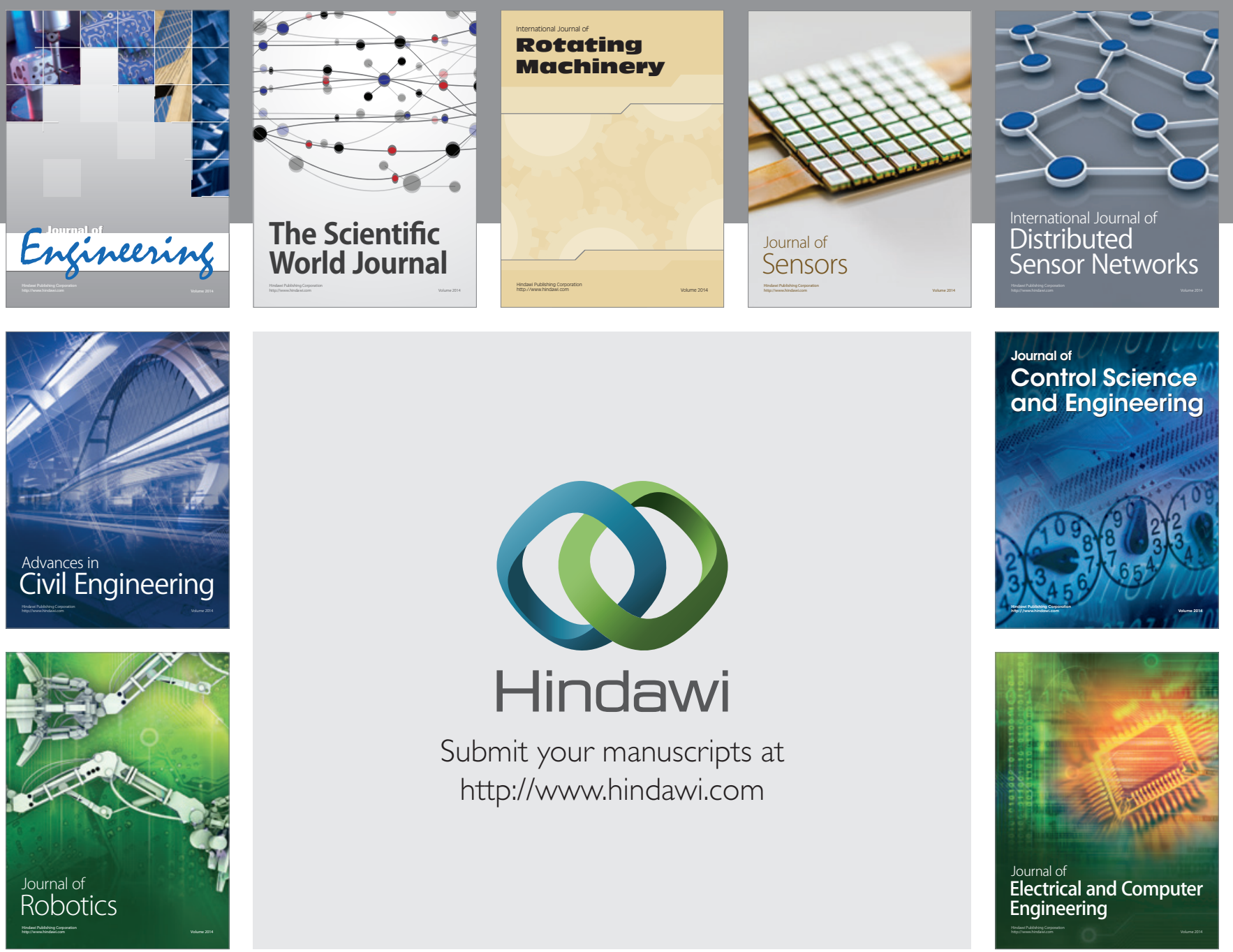

Submit your manuscripts at

http://www.hindawi.com
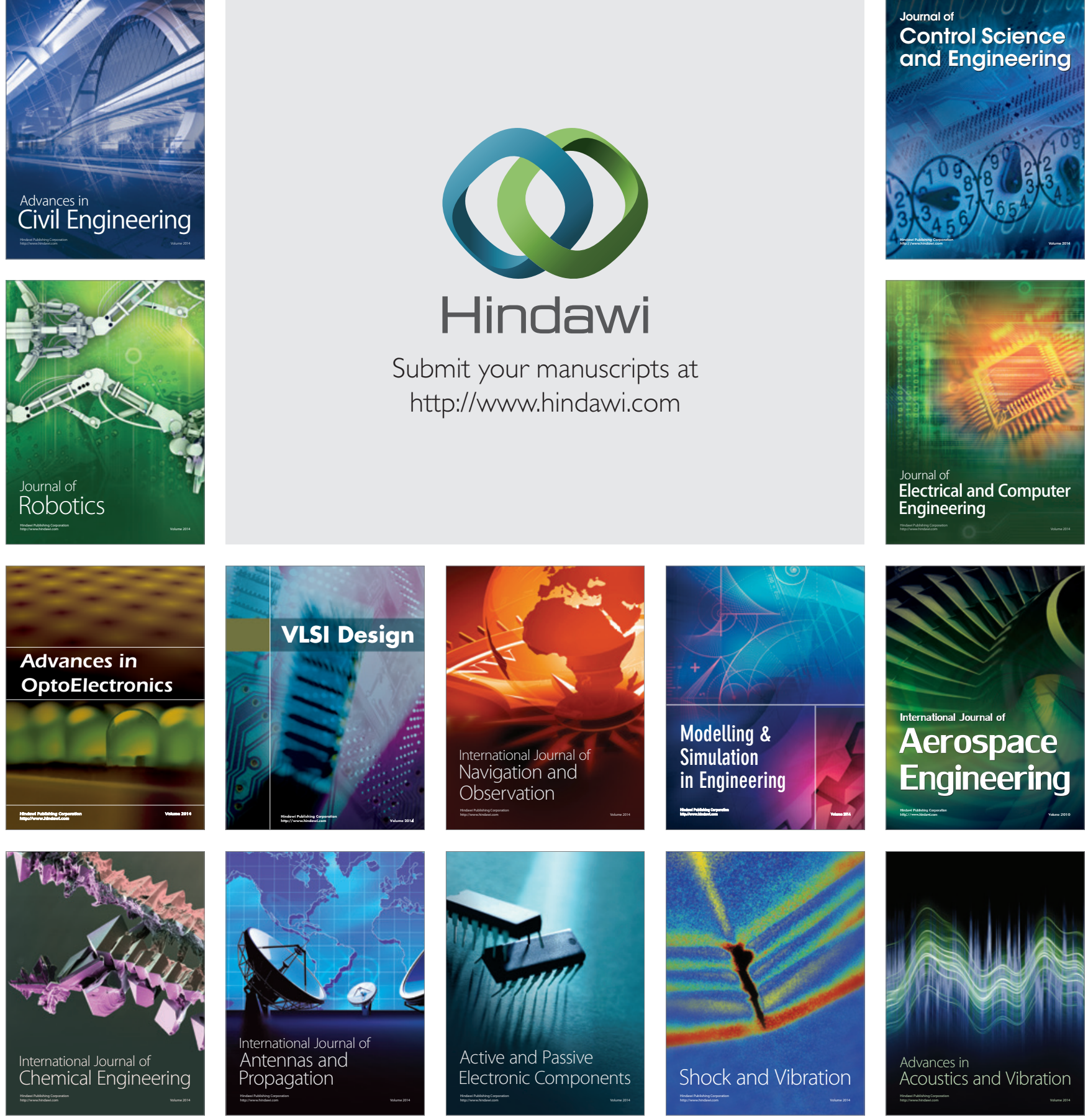